\title{
Network positions in active learning environments in physics
}

\author{
Adrienne L. Traxler®, ${ }^{1, *}$ Tyme Suda, ${ }^{1}$ Eric Brewe $\odot,{ }^{2}$ and Kelley Commeford $\odot^{3}$ \\ ${ }^{1}$ Wright State University, Department of Physics, Dayton, Ohio 45435, USA \\ ${ }^{2}$ Drexel University, Department of Physics, School of Education Philadelphia, Pennsylvania 19104, USA \\ ${ }^{3}$ Drexel University, Department of Physics, Philadelphia, Pennsylvania 19104, USA
}

(Received 15 April 2020; accepted 1 October 2020; published 27 October 2020)

\begin{abstract}
This study uses positional analysis to describe the student interaction networks in four research-based introductory physics curricula. Positional analysis is a technique for simplifying the structure of a network into blocks of actors whose connections are more similar to each other than to the rest of the network. This method describes social structure in a way that is comparable between networks of different sizes and densities and can show large-scale patterns such as hierarchy among positions. We detail one positional analysis method and apply it to class sections of Peer Instruction, SCALE-UP, ISLE, and Minnesota Model context-rich problems. At the level of detail shown in the blockmodels, most of the curricula are more alike than different, showing a late-term tendency to form coherent subgroups that communicate actively among themselves but have few interposition links. Initial position assignments tend to change from beginning to end of the term, but in cases where the initial assignment is stable, those students appear to become more connected to each other and to the largest network component. These trends in position structure and stability may be network signatures of active learning classes, but wider data collection is needed to investigate.
\end{abstract}

DOI: 10.1103/PhysRevPhysEducRes.16.020129

\section{INTRODUCTION}

Social network analysis (SNA) is a powerful toolkit for understanding the social structure of classrooms. It quantitatively describes the student-student interactions that are foundational to active learning. Network surveys are usable at a class-wide scale even with large enrollments, so they complement qualitative studies that can give deep detail on one or a few student groups. Most quantitative methods theoretically position individual traits (concept inventory scores, pass or fail outcomes, etc.) as the primary object of interest. However, in the intentionally collaborative and interdependent environment of active learning classrooms, this abstraction misses key ingredients of the learning experience. Network methods place equal theoretical emphasis on the actors and the patterns of connection among them. This dual focus makes networks an excellent lens for studying active learning.

This paper describes and applies a network technique, positional analysis, which is established in sociology but new to physics education research. Positional analysis gives

*adrienne.traxler@wright.edu

Published by the American Physical Society under the terms of the Creative Commons Attribution 4.0 International license. Further distribution of this work must maintain attribution to the author(s) and the published article's title, journal citation, and DOI. a mesoscale view of network structure, between finegrained details such as individual centrality and wholenetwork measures such as density. In this analysis, actors are grouped into positions by looking for similarities in their linking behavior, generating a small number of "positions" and a map of how they connect to summarize the terrain of the network.

We compare the social positions available in the classroom networks of four research-based introductory physics curricula. Various social network analyses have been done in physics education research (PER), but there is a scarcity of results that compare different institutions and class types using the same surveys and collection methods. Using the multisite data from the Characterizing Active Learning Environments in Physics (CALEP) project and developing a free implementation of a positional analysis algorithm, we are able to show broad similarities and a few striking differences in the social structures of these active learning environments in physics. Each set of network data for a given pedagogy comes from a single institution, so it is not possible to make claims such as "pedagogy $\mathrm{X}$ will lead to network structure Y." The enactment of a pedagogy is expected to differ between instructors and institutions, so much more data would be required for such a theory. However, this work aims to begin mapping the range of network structures that occur in highly active classrooms, with the long-term goal of helping researchers and instructors think more clearly about the structures of community in physics courses. 


\section{A. Social network analysis in physics education}

Physics education research and other discipline-based education research (DBER) fields have identified active learning as critical to effective instruction [1,2]. Though definitions of active learning are not all precise and aligned, they center interaction with others, particularly other students [3]. Attending to the structure of these interactions in classrooms and other learning environments has led to the use of social network analysis in PER [4] and DBER [5]. SNA has been used in PER in a variety of ways: to identify patterns of participation in an informal learning environment [6], to map conceptual flow in student interview responses or discussions $[7,8]$, to identify productive online forum discussions [9], to predict grades in current or future classes $[10,11]$, and to predict persistence in physics [12] and in degree programs [13].

Networks use relational data to quantify the connections between a set of entities. In the case of social networks, these entities (also known as nodes or actors) are people, and the structure of relations (edges or links) describes the structure of the social group along some dimension such as friendship or advice. To date, many studies of physics learning networks have focused on node-level traits and outcomes rather than overall network structure. Examples include mapping which students are most central, whether that is influenced by demographic variables, and how centrality interacts with outcomes like course success or persistence. Some results suggest that traditional passive lectures show sparse network structure and active learning networks are more connected [14], but these results are not universal [15], and are likely influenced by demographics, course-taking history, and other factors. There has been no systematic survey of what "normal" networks look like in active learning classrooms, or how to summarize a typical structure if one exists. Community detection algorithms offer one possible tool for this purpose.

\section{B. Community detection}

Identifying and describing communities within broader networks is an active research area in SNA [16]. In network analysis, a community refers to "groups of vertices which probably share common properties and/or play similar roles within the graph" [17]. Communities have been applied in a wide range of problems, such as understanding social divisions among humans and animals, mapping the structure of the World Wide Web, and studying protein interactions in cancerous cells [17]. In PER, community detection has been used to find clusters of related items on concept inventories [18,19], and to describe the number of student communities as a function of time in the semester $[15,20]$. If we wish to describe the structure of a network, communities are one important tool for doing so.

Knowing which students work together in a classroom can help to determine how information or other resources are likely to spread in the class, or whether interventions to build student connections have been successful. This information can be collected through classroom observations, but these are difficult to gather systematically. There is also value in having a student-derived account of the social structure. Community detection provides one possible answer to the question "what do the interactions in my interactive-engagement class look like?"

\section{Positional analysis}

Positional analysis can be classified as a community detection method (see discussion of "blockmodels" in Refs. $[17,21])$, because it looks for ways to group or block nodes so that they are more like their community members than they are the network as a whole. In many kinds of community detection, link density is the guiding principle, with algorithms finding node groups that are more connected to each other than to the surrounding network $[22,23]$. Other detection methods look for edges of high "betweenness," whose removal would disconnect groups of nodes [24]. In other cases, "random walk" algorithms look for minimal-information ways to describe the nested structure of nodes, communities, and networks [25]. These various approaches share a mathematical task of partitioning the network, ideally using only information contained in the network object. But any answer to the problem also contains theoretical assumptions about what network properties truly define a community.

Positional analysis comes from the sociological rather than the physics tradition of network analysis (Ref. [26] outlines this long-running theoretical divide). Rather than looking for the most tightly interlinked clusters of nodes, or those who could be separated by removal of key edges, it looks for "individuals who are similarly embedded in networks of relations" [27] (p. 348). These individuals form a discrete set of social blocks, and each block is a position. This level of abstraction helps us to think about the network in terms of broad dynamics. Is it hierarchical, with some positions dominant and others as hangers-on [28]? Are there substantial changes in the coherence or types of positions over time [29]? These questions are taken up much more often in the sociology tradition of network analysis. The methods in this paper come from that tradition, so they are promising tools for studying the social dynamics of classrooms.

Figure 1 shows a small example network, which might represent three levels of workers and managers in an organization. Positional analysis can pick out these structural levels as positions, even if not all the people in them are connected to each other (such as nodes D, E, F, and G). Positional analysis looks for similarity of connections and groups together nodes who have the greatest equivalence $[27,29,30]$. To find these groups, a positional analysis algorithm needs a definition of equivalence, a measure of similarity for describing how closely nodes approach equivalence, and a process for maximizing that measure. 


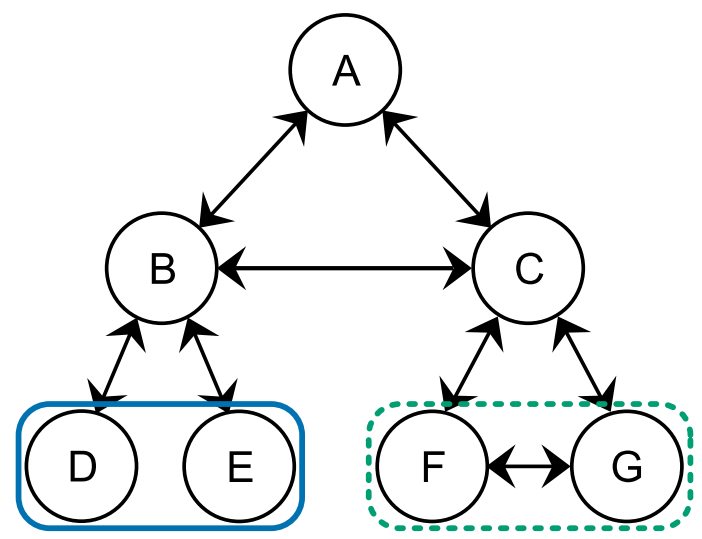

FIG. 1. A sample work network, where ties mean "meets weekly with." Positional analysis can identify layers of this example hierarchy, but structural equivalence will produce a more restrictive grouping than regular equivalence. By exact structural equivalence, only D/E (solid blue line) and F/G (dashed green line) are grouped together, with all other actors occupying singlemember positions.

The two most commonly used definitions of equivalence are structural and regular. Structural equivalence looks for the same ties to same others [27]. In Fig. 1, nodes D and E would be perfectly structurally equivalent (both connected only to node B), as would nodes F and $\mathrm{G}$ (both connected to $\mathrm{C}$ and to each other). On the other hand, regular equivalence looks for similar ties to similar others. In Fig. 1, nodes $\mathrm{B}$ and $\mathrm{C}$ might be regularly equivalent, and also nodes $\mathrm{D}$ through $\mathrm{G}$ (each of which connects to a node in the B/C group).

Regular equivalence is the more conceptually appealing definition in some ways - it can identify the three "levels" in Fig. 1, while perfect structural equivalence will not group any nodes beyond $\mathrm{D} / \mathrm{E}$ and $\mathrm{F} / \mathrm{G}$. However, regular equivalence is an ill-posed problem, with most networks having many possible partitions that all equally meet the definition of regular equivalence [31]. Structural equivalence also is less sensitive to missing data [32], which is a common concern in survey-based networks. For these reasons, and because it is mathematically more straightforward to define, we will use structural equivalence to look for network positions. Exact structural equivalence is rare to find in real networks, so methods to calculate it look for the closest possible match [27].

\section{Research questions}

We will address the following research questions:

1. What network positions emerge from the four different curriculum types?

2. What differences exist between early- and late-term network positions?

3. What major similarities or differences exist in network positions across learning environments?
Section II describes the four classroom settings, the data collection, and the details of the positional analysis algorithm. Section III summarizes network characteristics and positional analysis results for the early- and latesemester classroom surveys. Section IV discusses classroom and curricular implications of block structures. Section V revisits the research questions to give concluding notes, and Sec. VI outlines limitations and future work.

\section{METHODS}

\section{A. Positional analysis with CONCOR}

Positional analyses are done to partition a network into blocks, where block members have similar links to each other and to other nodes in the network. To do this, the network is first represented as an adjacency matrix, where a 1 in position $(i, j)$ indicates that node $i$ named a link to node $j$. Figure 2 shows the flow of positional analysis, beginning with this adjacency matrix step. Positional analysis permutes the adjacency matrix in order to group together similar nodes. When finished, the matrix will have blocks that are mostly filled with 1's and blocks that are mostly filled with 0 's, to the best extent possible for the data. How to carry out this permutation, and what final arrangement is the best possible one for the data, depends on the algorithm used.

\section{The CONCOR algorithm}

The convergence of iterated correlations (CONCOR) algorithm uses structural equivalence as its basis for sorting the adjacency matrix into blocks [30]. This means that it attempts to group nodes who have the "same ties to same others" - in other words, similar rows and/or columns of 1 's and 0's in the matrix. CONCOR uses the Pearson correlation to measure similarity between columns, iterating until all matrix entries converge to +1 or -1 . These \pm 1 values are used to separate the columns into two blocks: after convergence, all +1 columns are known to have more similar links to each other than they do to -1 columns. The process can be repeated to further divide each block. Thus, the structural equivalence criterion, together with the Pearson correlation, give both a success condition and a process for selecting the blocks.

The Pearson correlation between nodes $i$ and $j$ is defined as follows for multiple relations on an $N \times N$ adjacency matrix [27]:

$$
r_{i j}=\frac{\sum_{r=1}^{2 R} \sum_{k=1}^{N}\left(x_{i k r}-\bar{x}_{i \bullet}\right)\left(x_{j k r}-\bar{x}_{j \bullet}\right)}{\sqrt{\sum_{r=1}^{2 R} \sum_{k=1}^{N}\left(x_{i k r}-\bar{x}_{\bullet \bullet}\right)^{2}} \sqrt{\sum_{r=1}^{2 R} \sum_{k=1}^{N}\left(x_{j k r}-\bar{x}_{j \bullet}\right)^{2}}}
$$

for $i \neq k, j \neq k$. This condition on $i$ and $j$ excludes the diagonal of the matrix from the correlation-meaning that we do not require a node to link to itself for the purposes of 


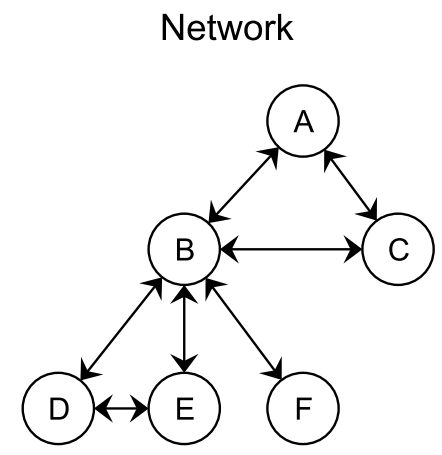

Adjacency matrix

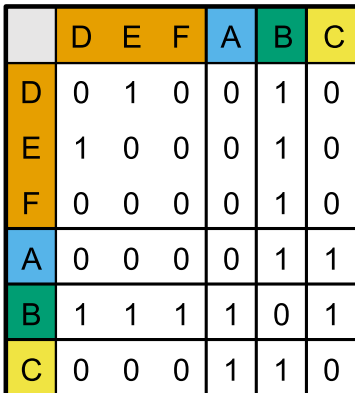

Blockmodel

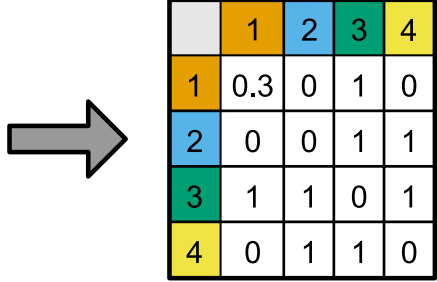

Density matrix

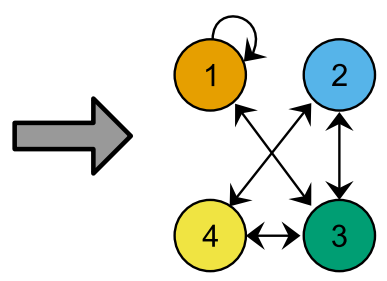

Reduced network

FIG. 2. Sample of blockmodel outputs. The network (top left) can be written as an adjacency matrix showing which nodes are linked (top right). The bottom row shows the blockmodeling products: a permuted adjacency matrix (left), the matrix showing each block density (center), and a reduced network where each diagonal block is treated as a node and inter- or intrablock links are present or absent according to a density threshold.

similarity. $R$ is the total number of relations (liking, animosity, etc.) between $N$ nodes. Our data is single relation, but CONCOR's ability to incorporate multiple link types is an advantage over many community detection methods. The symbol $\bar{x}_{i}$ is the mean of values in row $i$, and similarly, $\bar{x}_{\bullet i}$ is the mean of values in column $i$.

The original formulation of CONCOR proposed that correlations should be run on the columns of the adjacency matrix, though rows could be used instead [30]. Conceptually, this is the difference between looking for similarity in incoming ties (adjacency matrix columns) vs outgoing ties (adjacency matrix rows). We use the later version of the correlation given by Wasserman and Faust [27], which appends the transpose of the adjacency matrix to the original and then correlates the columns [28]. By this method, information from both outgoing and incoming ties is used to determine similarity.

When we began this work, we found one open-source implementation of CONCOR in R [33], the concoR package [34]. However, it was unable to reproduce known results for Krackhardt's high-tech managers network [27] (p. 379). On further scrutiny, we found that the concoR package only considered outgoing and not incoming ties (i.e., no transpose was included in the column correlation). Further, it used an unmodified form of the Pearson correlation that did not exclude self-ties. This form of the algorithm works similarly to the original version described in Breiger et al. [30], but is missing the improvements of Wasserman and Faust's more modern form. We could not find another fully functioning open source version of CONCOR, so we wrote our own. Our version, available in Ref. [35], incorporates information about both tie directions and successfully reproduces Wasserman and Faust's results on the high-tech managers network data [27] (Chap. 9).

One special case that the code must handle is isolates, nodes with no incoming or outgoing ties. We treat all isolates as structurally equivalent to each other, as they have the same connections (none) to all other nodes. The correlation loop in CONCOR fails for matrix columns of all zeros, so our code identifies and sets aside isolates before partitioning the other columns. Isolates are reported as a separate block at the end of the CONCOR-identified blocks. Aside from this special case, the order of the blocks is not significant. Our implementation works for an arbitrary number of splits (assuming there are structurally inequivalent nodes to separate) and allows for an arbitrary number of different relations to be included. A single measured network (for example, meaningful interactions in week 1) is a single relation, but two time points for the same network could be construed as multiple relations and used to show the time development of positions [29]. In the results below, we calculated single-relation CONCOR 
TABLE I. Survey response rates and proportions of observed and unobserved ties for the sections analyzed in this paper.

\begin{tabular}{|c|c|c|c|c|c|c|c|c|}
\hline Site & Time & $\begin{array}{l}\text { Nodes } \\
(n)\end{array}$ & $\begin{array}{l}\text { Responded } \\
\quad(1-m)\end{array}$ & $\begin{array}{l}\text { Response } \\
\text { rate }\end{array}$ & Observed & $\begin{array}{l}\text { Partially } \\
\text { observed }\end{array}$ & $\begin{array}{l}\text { Missing } \\
(\mathrm{NR}-\mathrm{R})\end{array}$ & $\begin{array}{c}\text { Missing } \\
(\mathrm{NR}-\mathrm{NR})\end{array}$ \\
\hline Peer Instruction & Early & 116 & 81 & $70 \%$ & 0.49 & 0.21 & 0.21 & 0.09 \\
\hline Peer Instruction & Late & 116 & 76 & $66 \%$ & 0.43 & 0.23 & 0.23 & 0.12 \\
\hline SCALE-UP & Early & 71 & 29 & $41 \%$ & 0.16 & 0.25 & 0.25 & 0.35 \\
\hline SCALE-UP & Late & 71 & 41 & $58 \%$ & 0.33 & 0.25 & 0.25 & 0.18 \\
\hline ISLE & Early & 27 & 14 & $52 \%$ & 0.26 & 0.26 & 0.26 & 0.22 \\
\hline ISLE & Late & 27 & 16 & $59 \%$ & 0.34 & 0.25 & 0.25 & 0.16 \\
\hline Context-rich problems & Early & 48 & 27 & $56 \%$ & 0.31 & 0.25 & 0.25 & 0.19 \\
\hline Context-rich problems & Late & 48 & 20 & $42 \%$ & 0.17 & 0.25 & 0.25 & 0.36 \\
\hline
\end{tabular}

blocks for each network sample (week 1 and week 10 for each class).

\section{Treatment of missing data}

Missing data are a common problem in network analyses, and can bias results by altering the network structure being studied. In real data, it is hard to know whether nodes are missing entirely at random, or if there is a systematic pattern to nonrespondents. There is some evidence that missing responses are more likely for people who are weakly connected to the network [36]. However, effects that lead to missingness can vary based on population and survey methods. For example, students who are often absent from class may miss an in-person survey, or active but very busy people might fail to respond to an email invitation [37]. Without detailed follow-up, it is difficult to say what effects might dominate in this case, so we will be guided by results based on random missingness [38].

There are several common methods for handling this issue: reporting rates but otherwise ignoring missing data, restricting the network to ties between respondents, imputing missing data, and correcting via exponential random graph models $[38,39]$. Huisman [39] simulated the effects of missing data and several imputation methods on a friendship network of 50 actors, which is in the same size and density range as the classroom networks in our study. He found that for directed networks with more than small amounts (30\%) of missing data, imputing the unobserved ties gave more biased estimates of network statistics than ignoring missingness for most measures. In another study, Žnidaršič et al. [32] simulated the effects of missing data on blockmodels of small networks. They found that overall, restricting to complete cases (dropping nonrespondents and any reported links to them) caused the least distortion in identifying network positions.

Imputing data is also not advised if the respondents and nonrespondents significantly differ in demographic traits or linking behavior [40]. Claims about linking behavior can be made by comparing the fraction of links received by respondents and nonrespondents. However, claims about demographic similarity would require additional data that was unavailable to us. After reviewing these results, we opted to avoid imputation and report results for two versions of the networks: all observed ties (ignoring missingness in the data), and complete cases only (removing nonrespondent nodes and any links to them). The allties results are given in the main text, and the complete case results are in the Supplemental Material [41].

\section{Response rates}

We chose sections for analysis with the highest possible response rates while still representing a range of curricula. If $n$ is the number of possible actors (students in a section) and $m$ is the number who did not respond to the survey, then the actor response rate is $(1-m / n)$ [32]. The $(n-m)$ respondents will have $(n-m)(n-m-1)$ measured ties among each other with values of either 0 or 1 . These are the fully observed ties, and their proportion to all possible ties in the network is $(n-m)(n-m-1) / n(n-1)$. Partially observed ties connect respondents and nonrespondents, and their proportion of the total is $(n-m) m / n(n-1)$. There are two categories of missing ties: those from nonrespondents to respondents [fraction $m(n-m)$ / $n(n-1)$ ] and those between nonrespondents [fraction $m(m-1) / n(n-1)]$. Table I shows the sections chosen for analysis and their proportions of observed and unobserved ties. The best-case data displayed here is for Peer Instruction $(66 \%-70 \%$ of ties partially or completely reported) and the worst case is SCALE-UP (41\%-58\% of ties partially or completely reported).

\section{Outputs}

The first iteration of CONCOR on a matrix will divide it into two sets of columns, representing two groups of nodes whose edges are more similar to each other than to the remaining nodes. Running CONCOR again will further divide each of those groups into two subgroups. The algorithm stops after it has reached a specified number of "splits." The appropriate number of splits depends on the application and often must be found experimentally. In the case of our data, one split tended to produce large positions, which were not very informative. Three or more splits often 
failed, because the algorithm will not converge if it tries to divide a completely connected group. We report results for two CONCOR splits for all sections.

The outputs of positional analysis, whether by CONCOR or another algorithm, are

- A blockmodel, which permutes the rows and columns of the original adjacency matrix to partition the network into positions.

- An image matrix, which shows only the edge density within each block.

- A reduced network, which thresholds values in the image matrix to 0 or 1 and plots the result as a network.

Each level of output further condenses the information in the original network, with the reduced network plot showing a graphical summary of the network positions and the connections between them. For calculating the density matrix, each block (whether on- or off-diagonal) has a density equal to its number of 1's divided by the number of possible links in it (number of rows $\times$ number of columns in the block). Self-ties are omitted, so for on-diagonal blocks with $n$ nodes, the number of possible ties is $n(n-1)$.

Figure 2 shows the stages of the process for a fictional network. We will show the original network, the blockmodel, and the reduced network for each class section and time point.

\section{B. Data and class context}

Project sites were chosen for having established, highfidelity implementations of research-based curricula in introductory physics. A researcher visited each site to take classroom observation data, discussed in another paper [42]. In the first and tenth weeks of the term, a survey was distributed to students through Qualtrics which asked them to respond to the following prompt: "Please choose from the list of people that are enrolled in your physics class the names of any other student with whom you had a meaningful interaction in class during the past week, even if you were not the main person speaking." Students were then given a class roster of names to choose from. This form of the survey prompt has been used and validated in previous classroom network studies [12]. Providing a roster and a specific time frame in network surveys boosts recall and reliability of the question (that is, how consistently different students interpret what they are asked) [43]. Survey invitations were emailed by the researcher, and reminder emails were sent after 1 to 3 days.

The curriculum types shown in this paper are Peer Instruction, SCALE-UP, ISLE, and Minnesota Model context-rich problems. The number of sections surveyed varied from 1 (SCALE-UP) to 39 (ISLE), depending on the size of the school and the teaching schedule for introductory physics. From each curriculum type, the section with the best combined week 1 and week 10 response rate was used in this analysis. Brief summaries of each institution and class context are below; additional details appear in Commeford, Brewe, and Traxler [42].

\section{Peer Instruction}

Peer Instruction [44] was developed to allow for active learning in large lecture halls, often with immobile seats. The instructor poses questions with carefully chosen distractors to elicit misconceptions and spark debate. Students individually "vote" their answers (using electronic clickers, paper cards, or other means), and the instructor evaluates the answer distribution. In many cases it is useful to have students discuss their answer with one or two neighbors, then the class votes again. Peer Instruction has a long record of evidence for student learning gains [45] and is one of the most widely adopted research-based teaching strategies for new physics faculty [46].

Peer Instruction data were taken from a large, primarily residential private university in the northeastern United States, with a Carnegie classification of very high research activity. The 2017-2018 student body was 24190 (64\% undergraduate). The racial and ethnic demographics of the students at the university level in Fall 2018 were 8\% African American, 15\% Asian, 6\% Hispanic, 53\% White, $3 \%$ more than one race, $<1 \%$ Native American and Pacific Islander.

\section{SCALE-UP}

Student-Centered Activities for Large Enrollment Undergraduate Programs (SCALE-UP) is a studio format classroom type where lecture and lab time are combined and computers are on hand to help with activities [47]. Students work in small groups, typically three groups of three students to a table, on a variety of "tangible" or "ponderable" activities, labs, or other problem types. Class is usually divided into short segments of 5-15 min interspersed with discussions, and lecture occurs among these segments to synthesize and organize the activities.

SCALE-UP data were taken at a large, primarily residential public university in the midwestern United States, with a Carnegie classification of high research activity. The 2016-2017 student body was 14432 (86\% undergraduate). The racial and ethnographic demographics of the students at the university level in Fall 2018 were 3\% Black or African American, 2\% Asian, 2\% Hispanic, $82 \%$ White, $3 \%$ more than one race, $1 \%$ American Indian/ Alaskan, $<1 \%$ Hawaiian/Pacific Islander.

\section{ISLE}

Investigative Science Learning Environment (ISLE), is a curriculum that can be implemented in laboratory sections or (ideally) across the lecture, lab, and recitation components of a class. The units guide students through cycles of observing phenomena, finding patterns, and developing theories to test predictions [48]. There is an emphasis on 
TABLE II. Network descriptive statistics for the classes analyzed: number of nodes $(N)$ and edges $\left(N_{e}\right)$, fraction of named ties that were reciprocated, network density, average degree, and transitivity. For the last three values, the standard error of the final digit is given in parentheses.

\begin{tabular}{lccccccc}
\hline \hline Site & Time & $N$ & $N_{e}$ & Reciprocity & Density & Average degree & Transitivity \\
\hline Peer Instruction & Early & 94 & 130 & 0.63 & $0.015(3)$ & $2.8(3)$ & $3.3(3)$ \\
Peer Instruction & Late & 97 & 159 & 0.70 & $0.017(3)$ & $0.23(9)$ \\
SCALE-UP & Early & 56 & 68 & 0.60 & $0.022(6)$ & $2.4(3)$ & $0.23(9)$ \\
SCALE-UP & Late & 66 & 176 & 0.65 & $0.041(7)$ & $0.3(1)$ \\
ISLE & Early & 20 & 19 & 0.50 & $0.05(2)$ & $0.9(5)$ & $0.9(9)$ \\
ISLE & Late & 24 & 47 & 0.93 & $0.09(2)$ & $0.3(2)$ \\
Context-rich problems & Early & 40 & 48 & 0.42 & $0.031(8)$ & $2.4(3)$ & $0.6(2)$ \\
Context-rich problems & Late & 41 & 96 & 0.62 & $0.06(2)$ & $4.7(6)$ & $0.2(1)$ \\
\hline \hline
\end{tabular}

coordinating multiple representations, and the curriculum uses a cognitive apprenticeship model to help students learn about the nature of science as they develop their physics ideas [48]. The section shown in this paper was a lab-only implementation.

ISLE data were taken from a large, primarily residential public university in the northeastern United States, with a Carnegie classification of very high research activity. The 2018-2019 student body was 42828 (78\% undergraduate). The racial and ethnographic demographics of the students at the university level in Fall 2017 were 8\% African American, 22\% Asian, 12\% Hispanic, 40\% White, 3\% more than one race, $13 \%$ international, and $2 \%$ other (including Native American and Pacific Islander).

\section{Context-rich problems}

The Minnesota Model for Large Introductory Courses [49] sets out a plan for coordinating the elements and people involved in introductory physics courses. The most widely known aspect of the model, published and disseminated in venues including the AAPT, AAS, and APS New Faculty Workshops, is the use of cooperative group problem solving with context-rich problems. Since this aspect is often used as a shorthand for the larger framework, we will use "context-rich problems" to refer to this curriculum in the remainder of the paper.

Context-rich problems, also called cooperative problem solving, is a method often used in recitation sections attached to a lecture course [50,51]. Students work in small groups on problems that are framed less straightforwardly than typical textbook problems ("context rich"), where deciding what quantities must be solved for is often a necessary step. The solution process is explicitly structured to follow expert problem-solving habits [50,51]. Groups have been shown to outperform their highest-scoring individual members, as well as a parallel class taught in a traditional lecture style without this problem-solving framework [51].

Context-rich problems data were taken from a very large public associate's college in the western United States.
The 2017-2018 student body was 34642 (100\% undergraduate). The racial and ethnographic demographics of the students in Fall 2017 were 2\% African American, 5\% Asian, $33 \%$ Latino, $50 \%$ White, $6 \%$ more than one race, $3 \%$ unknown, and $1 \%$ Native American and Pacific Islander.

\section{RESULTS}

\section{A. Network descriptions}

Table II shows descriptive statistics for the networks discussed in this paper. This includes number of nodes and edges (including nonrespondents who were named by survey takers), reciprocity among respondents, network density, average degree, and clustering coefficient. The Supplemental Material [41] gives the same results for the complete-cases networks, where only survey respondents and links among them are retained.

Reciprocity is calculated as the probability that for each directed edge, the opposite-pointing edge is also in the graph. Density, average degree, and transitivity are three related ways of describing how well connected a network is. Density is the fraction of present to possible ties, and for a directed network, is

$$
\rho=N_{e} / N(N-1),
$$

where $N$ is the number of nodes and $N_{e}$ is the number of edges. Because density scales as $1 / N^{2}$, it is misleading to compare densities between networks of different sizes (larger networks will tend to have lower density). The last two measures in the table give estimates of connectivity that are comparable across networks of different sizes. Average degree describes nodes' number of outgoing and incoming connections, and is calculated by averaging the row and column sums of the adjacency matrix. Transitivity, or clustering coefficient, is the probability that any two nodes who have a neighbor in common will themselves be connected ("the friend of my friend is also my friend") [21]. All statistics are calculated in R [33] using the igraph package [52]. 
Snijders and Borgatti [53] outline a bootstrap process for estimating the variability in network statistics: resample the network many times, recalculate the statistic using each sampled network, and then calculate the standard deviation of all the sampled statistic values. This estimates the standard error for the measured value of the statistic. Table II includes standard errors for density, average degree, and transitivity using the method of Ref. [53] with 1000 bootstrap trials.

The Peer Instruction network shows only a marginal increase in density over the semester, but in the other three classes, the week 10 density roughly doubles from its week 1 value. A similar pattern exists for average degree. For transitivity, the Peer Instruction and context-rich problems values stay relatively steady, while the other two class types increase.

In the Peer Instruction and SCALE-UP sections, the majority of links are reciprocated in both week 1 and 10. In ISLE, the week 1 reciprocity rate is lower at $50 \%$, but in week 10 is the highest, at $90 \%$. Context-rich problems show a smaller increase, with the lowest starting reciprocity but a week 10 value comparable to Peer Instruction and SCALE-UP. There is no clear pattern connecting class size with reciprocity, average degree, or transitivity.

\section{B. Peer Instruction}

The top row of Fig. 3 shows the network diagrams for week 1 and week 10 of a Peer Instruction section. The larger circles are survey respondents, while smaller circles are named nonrespondents. The links are directed, so only a double-headed arrow between two nodes is reciprocal. Nodes are colored by their position (the partition they were assigned by CONCOR, which can change from week 1 to week 10). In week 1 , the giant component is comprised of the first two positions (orange and light blue nodes), the fourth position (yellow), and some nodes in the third position (green). (The "giant component," or largest set of connected nodes, is of interest because it is the largest information-sharing pool in the network [21].) Largely, however, green nodes are in smaller clumps not linked to the giant component. From week 1 to week 10, more of the nodes become linked to the giant component, but there are still several smaller groups and seven isolates (compared to 12 in week 1).

The middle row of Fig. 3 shows the blockmodel for each week, which is the adjacency matrix permuted to group together CONCOR positions in blocks. Each square on the plot would be a 1 rather than a 0 if the adjacency matrix was plotted as numbers. The blockmodel plots contextualize the change in reduced networks from early to late semester. In week 1 , the first two positions (the top two on-diagonal blocks, orange and light blue) are notably higher density than others. The second two positions (green and yellow) show CONCOR's attempt to segregate a more diffuse collection of links. In week 10, the on-diagonal blocks have a more similar density.

The bottom row of Fig. 3 shows the corresponding reduced networks. Each node in a reduced network represents all nodes of that color from the original network (and the diagonal block of that color on the blockmodel). Four of the positions have substantial communication among themselves both early and late semester, represented by the selfconnected "loop" edges on the reduced network. Early in the semester, there appears to be more cross-talk between positions, with one additional link between positions four and three, and another between positions one and two.

\section{SCALE-UP}

Figure 4 shows the network diagrams, blockmodels, and reduced networks for week 1 and week 10 of the SCALEUP section. There is a significant increase in link density from week 1 to week 10 , and all late-term survey respondents reported at least one meaningful interaction (no isolates exist on the week 10 graph). In week 1, only $48 \%$ of the nodes are in the giant component, which includes all of positions one and two (orange and light blue) and some of position three (green). Position four (yellow) is a fairly coherent subgroup of seven nodes. In week 10, the giant component has grown to contain $88 \%$ of the nodes. CONCOR divides most of the giant component into three subgroups that are fairly coherent among themselves. The fourth position (yellow) is a collection of relatively peripheral nodes.

In the week 1 blockmodel plot, there were three small positions - two of them fairly coherent-and a large group of nodes with less interconnection. This group (third diagonal block) corresponds to the green nodes on the graph, many of whom were named by survey respondents but did not take the survey themselves (and thus links they may have are unknown). By week 10, the positions are more uniform in size, and have higher internal link density. On the reduced graphs, both early and late term, all positions except the isolates (dark blue) talk among themselves. In week 1, there is also an appreciable amount of interaction from position two to one (light blue $\rightarrow$ orange), but this is gone in week 10 .

\section{ISLE}

Figure 5 shows the plots for week 1 and week 10 of the ISLE section. In week 1, the network is low density, with fewer than half of the nodes in the giant component. In week 10, that component is larger ( $71 \%$ of nodes), and is now divided between three of the four CONCOR-identified positions.

The network and blockmodel diagrams for week 1 show some of the differences between positional analysis and most community-funding algorithms. Light blue nodes connect to orange and vice versa, but are only adjacent to nodes in their own position in one case (the single light 


\section{Peer Instruction Early}

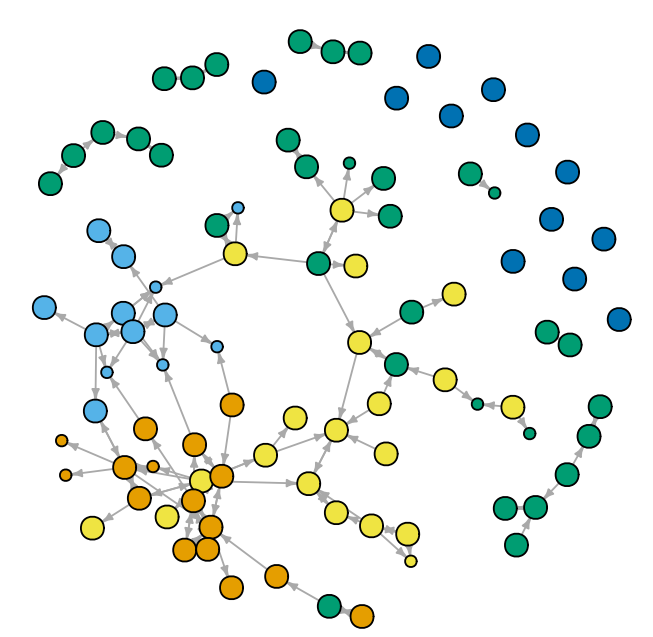

Peer Instruction Late

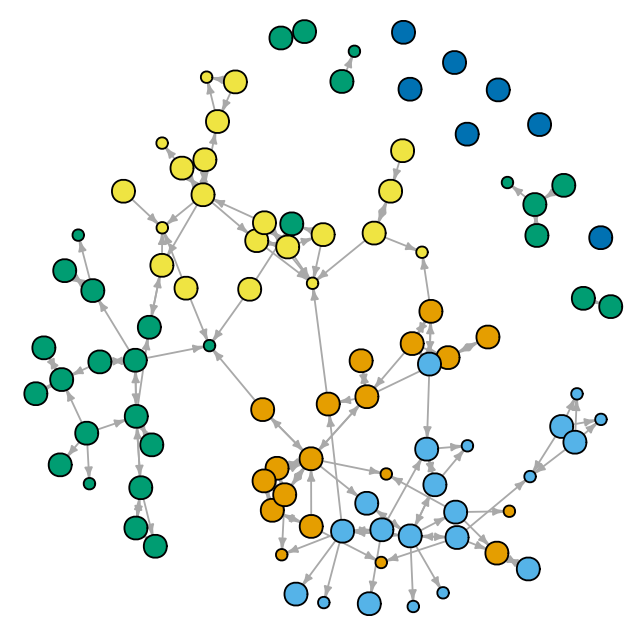

(a)
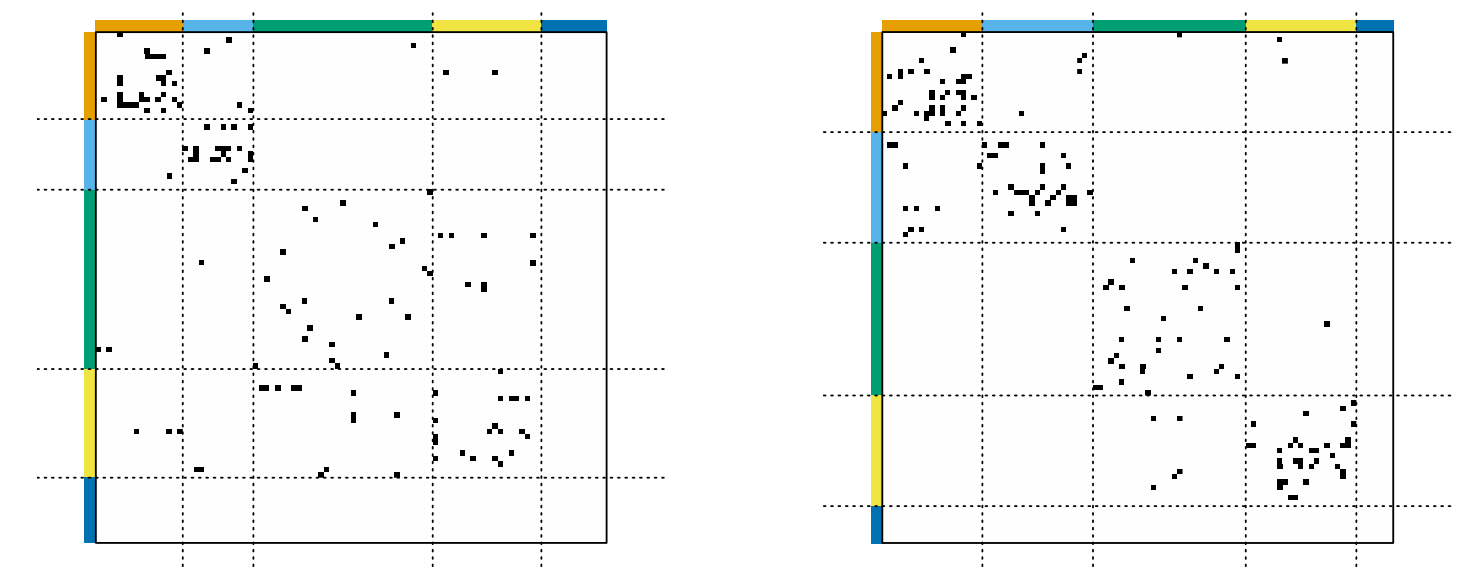

(b)<smiles>O[Os]</smiles>

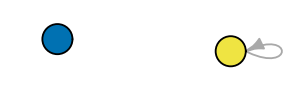

(c)
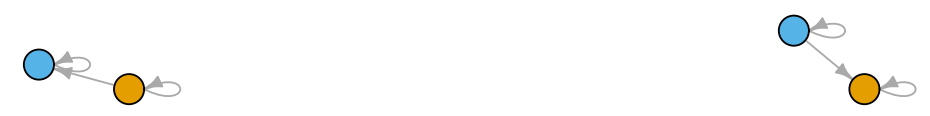

FIG. 3. (a) Network diagrams for week 1 (left column) and week 10 (right column) Peer Instruction section. Smaller circles show students who were named but did not take the survey. Nodes are colored by CONCOR partition, and indicate the same nodes in the blockmodel and reduced network below. (b) The corresponding blockmodel. Each link from the survey is a black square. The dotted lines mark the CONCOR group partitions. Colors along the sides mark which blocks of nodes belong to each partition. (c) Reduced network diagrams. Each circle represents a position from the blockmodel and stands for all of the nodes of the same color in the network diagram. Links in the reduced network plots show connections within and between positions and come from applying a density threshold to the blockmodel.

blue $\rightarrow$ light blue link on the week 1 blockmodel). Community detection routines do not generally "skip" in-between nodes to cluster two or more nodes that do not link to each other.
On the reduced networks, the week 10 diagram shows the relatively clean separation of the network into subcomponents. This separation, visible on the network diagram, translates into a reduced network with four "island" 
SCALE-UP Early

(a)

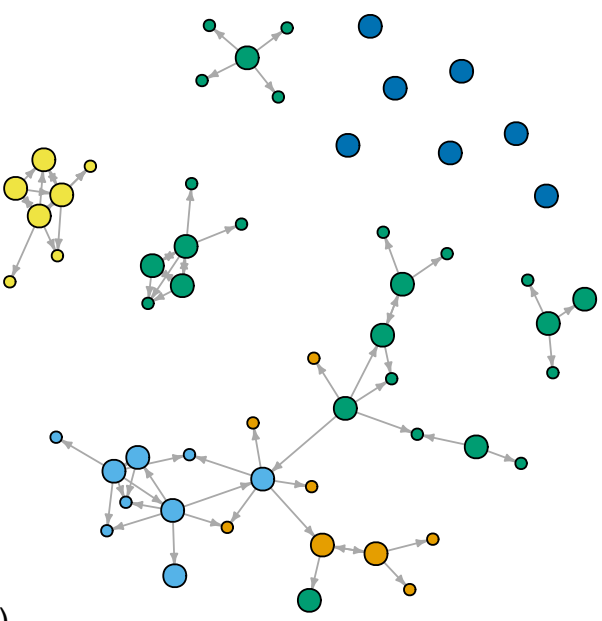

SCALE-UP Late

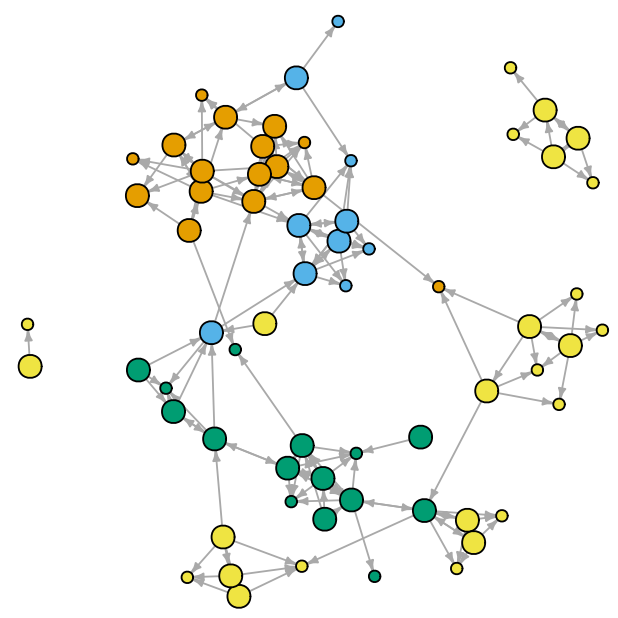

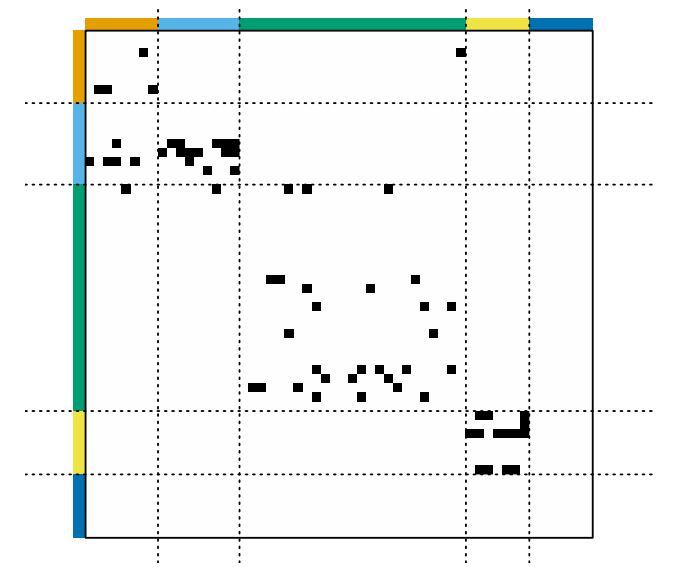

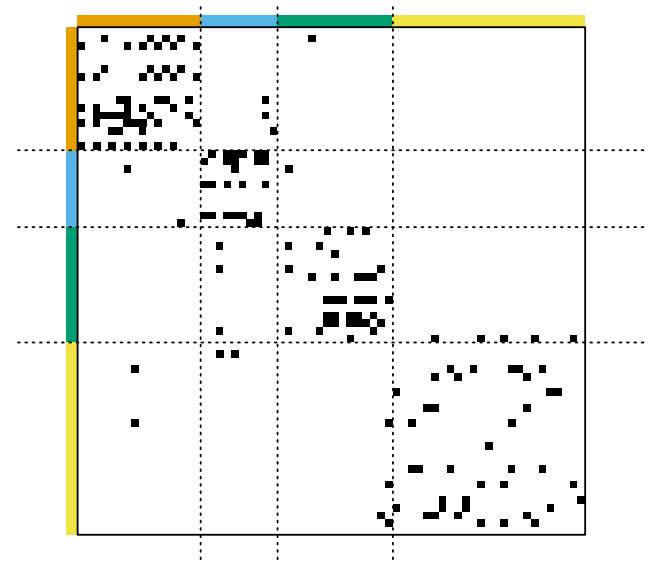

(b)

(c)
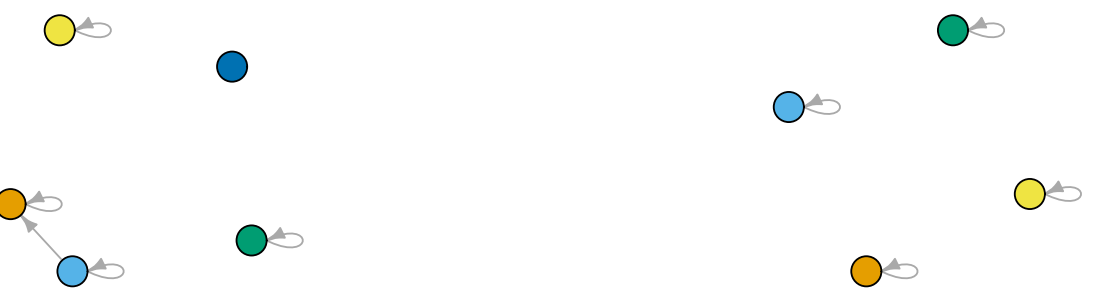

FIG. 4. (a) Network diagrams, (b) blockmodels, and (c) reduced network diagrams for SCALE-UP section. Smaller circles on the network diagrams are students who did not take the survey but were named by respondents. Nodes are colored by CONCOR partition, and dotted lines on the blockmodel plots show the same partition.

positions. Students named other people from their position on the survey, but rarely indicated links between blocks.

\section{E. Context-rich problems}

Figure 6 shows the network diagrams, blockmodel plots, and reduced networks for week 1 and week 10 of the context-rich problems section. In week 1 (and as in other early-term classes), the network is relatively sparse, with a loosely connected giant component, two island groups, and several isolates. The week 10 network has doubled in density, with all respondents in the giant component and a nearly $50 \%$ increase in reciprocity in naming interactions. The reduced network has the highest number of links between positions of any class analyzed, both early and late in the semester. 
ISLE Early

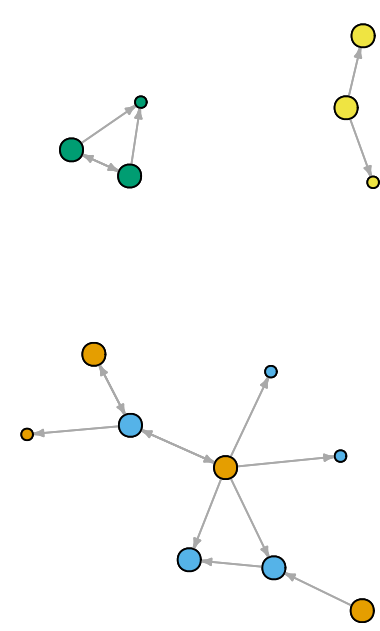

ISLE Late

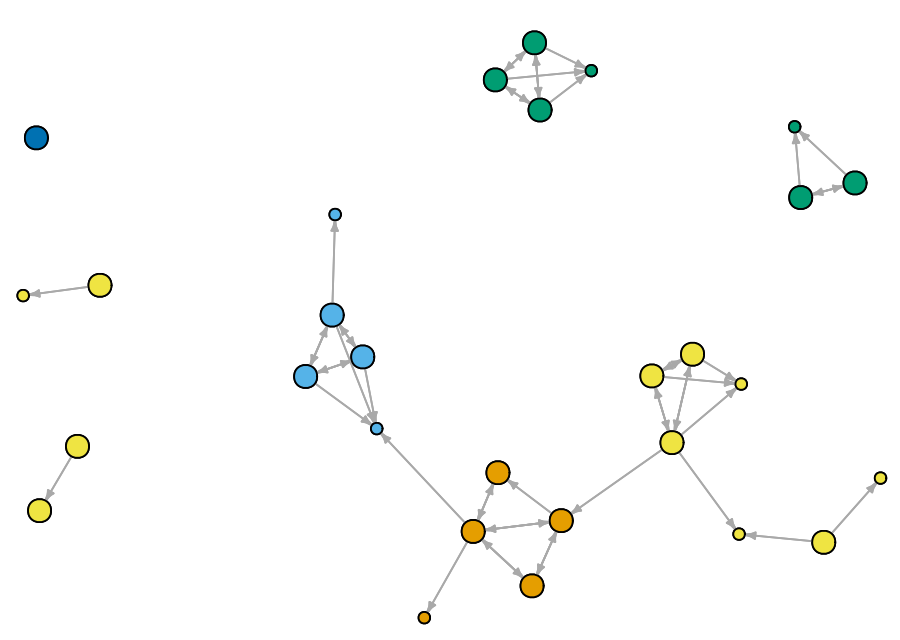

(a)
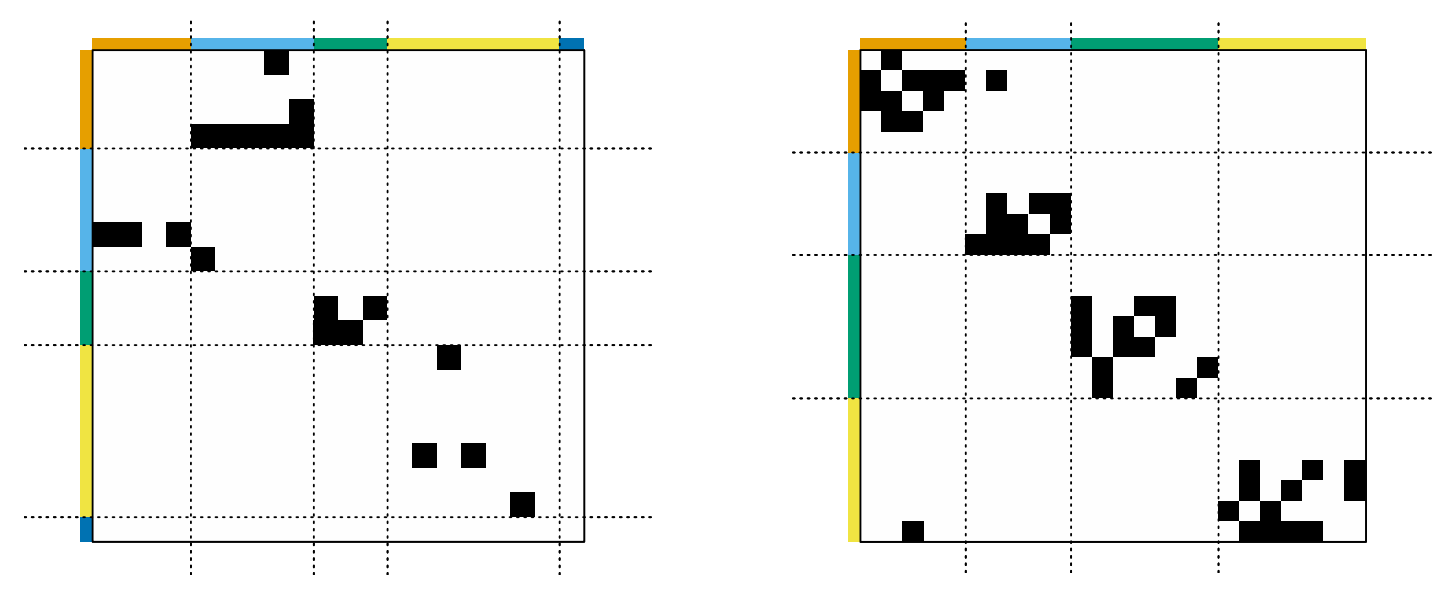

(b)

(c)
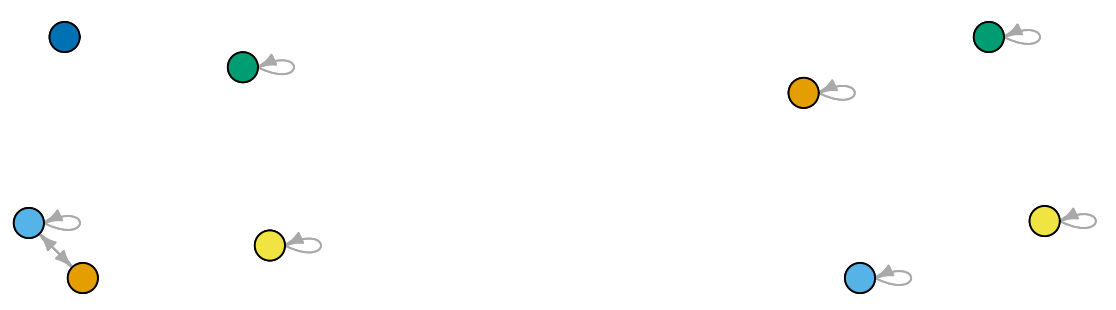

FIG. 5. (a) Network diagrams, (b) blockmodels, and (c) reduced network diagrams for ISLE section. Smaller circles on the network diagrams are students who did not take the survey but were named by respondents. Nodes are colored by CONCOR partition, and dotted lines on the blockmodel plots show the same partition.

The low response rate in week 10 appears to cause some artifacts in the CONCOR blocking. In particular, position one (orange) is dominated by nonrespondents, and likely would have more outgoing links if those people had taken the survey. The other positions, particularly three and four, have a lesser version of the same problem.

\section{F. Time development of blocks}

Figure 7 shows alluvial diagrams [54] for all four sections. Alluvial diagrams show how the categorization of a data set changes from one time point to another. Applied to this data, they show to what extent students stay in the same CONCOR block from week 1 to week 10 of the 


\section{Context-Rich Problems Early}

\section{Context-Rich Problems Late}
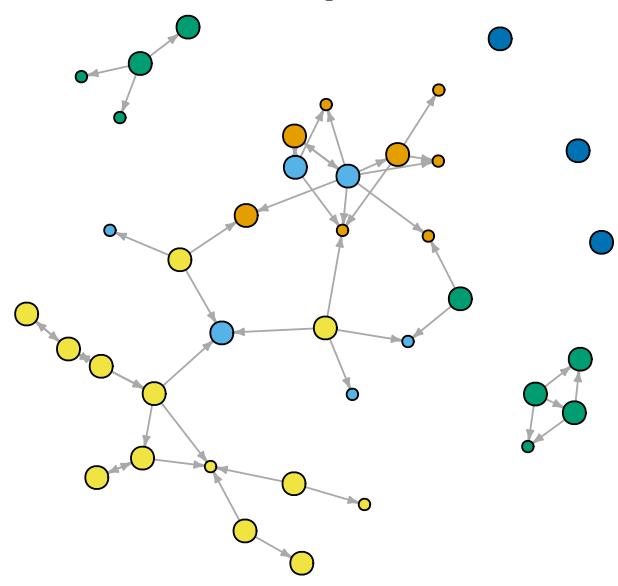

(a)
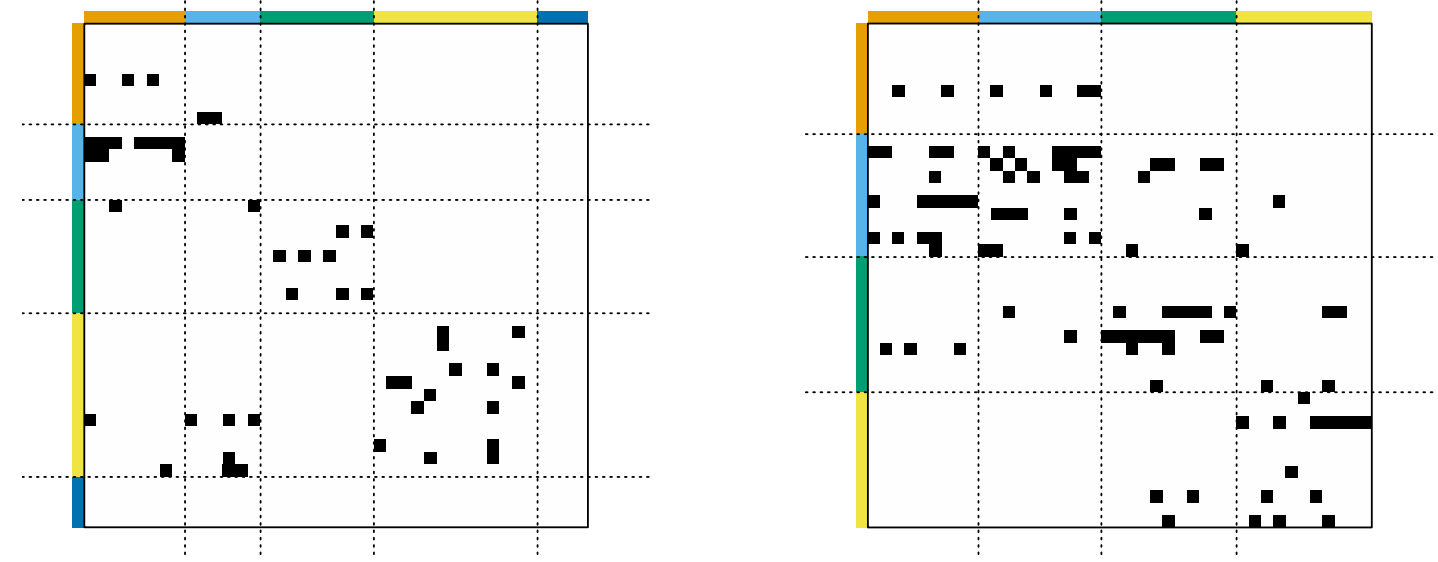

(b)

(c)

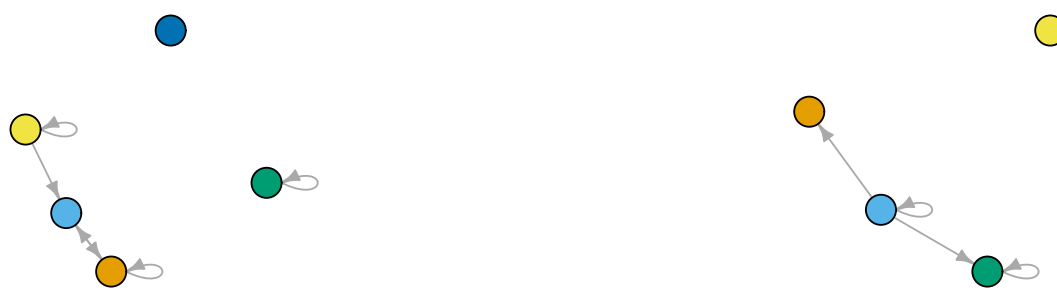

FIG. 6. (a) Network diagrams, (b) blockmodels, and (c) reduced network diagrams for context-rich problems section. Smaller circles on the network diagrams are students who did not take the survey but were named by respondents. Nodes are colored by CONCOR partition, and dotted lines on the blockmodel plots show the same partition.

term. Each panel in Fig. 7 shows the week 1 block assignments in the first column, and the week 10 assignments for the same people in the second column. The portion of each column coded "missing" is students who were not present in that survey.
In the Peer Instruction section, a substantial portion of students in blocks 1, 2, and 3 retained their week 1 positional assignment, with smaller portions of blocks 4 and 5 also staying. Comparing with the network diagrams in Fig. 3, the "core" of the giant component was largely 

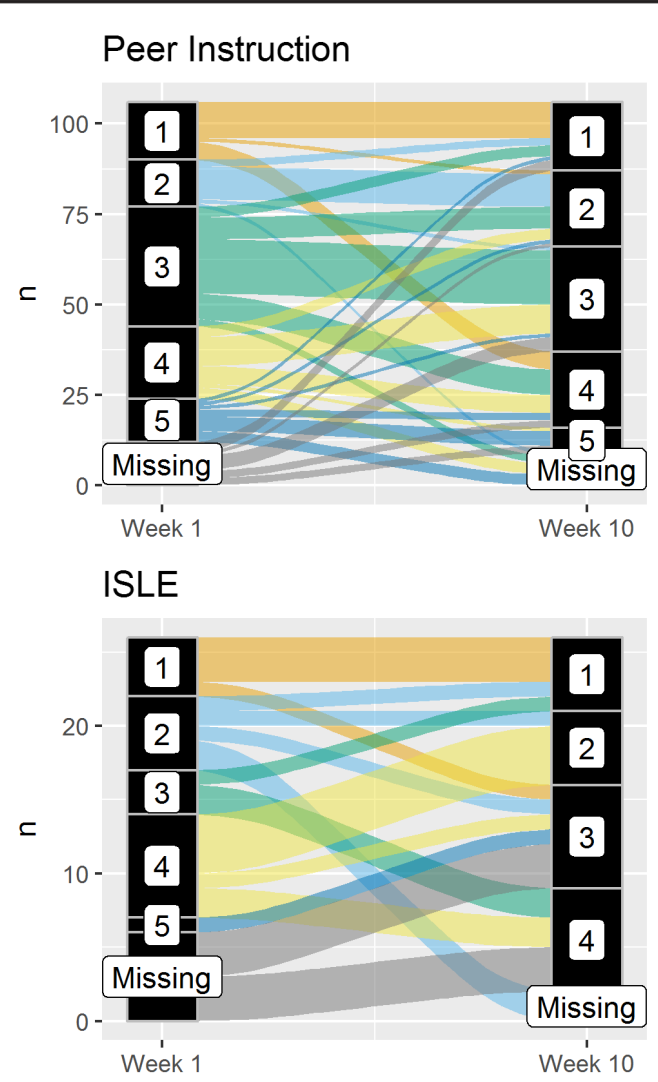

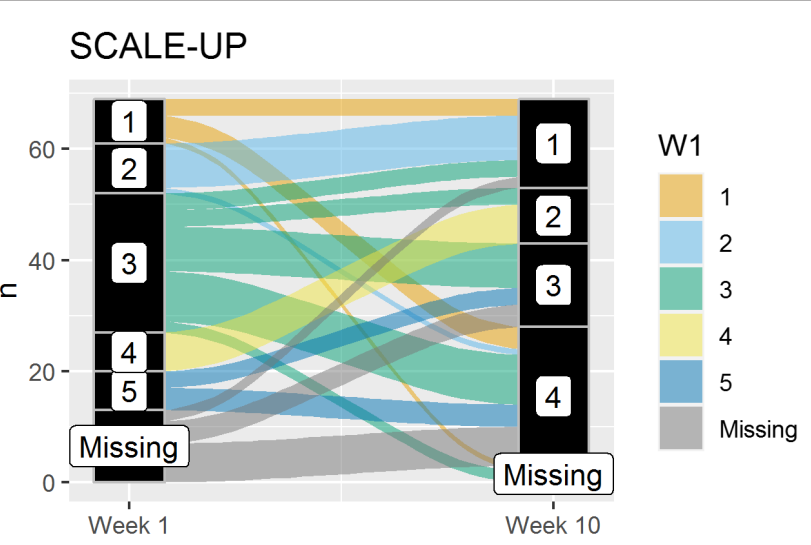

\section{Context-Rich Problems}

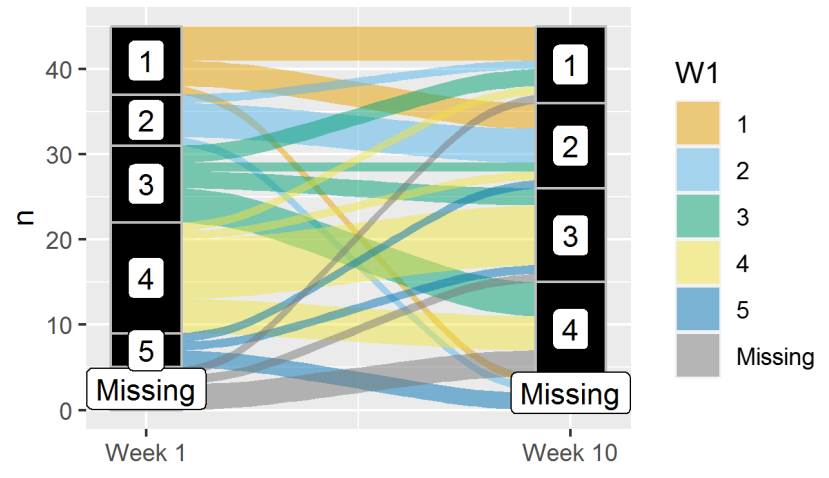

FIG. 7. Each diagram shows students' week 1 CONCOR block assignment in the first column, and their week 10 assignment in the second column. Colors match the original block assignment to help with visually following the flow. The missing label indicates students who were not present (either as survey takers or named) in that week's data.

occupied by positions 1 and 2 and was relatively stable. Position 4 (yellow) was also in the giant component but was more tenuously connected (both among itself and to the giant component), and was also the most fragmented in the week $1 \rightarrow$ week 10 alluvial diagram.

The SCALE-UP alluvial diagram in Fig. 7 shows some apparent shifting of positions that is actually an artifact of the algorithm. In particular, positions 2 and 4 stay coherent from week 1 to week 10 , but their numbering is different in week 10, so that the light blue nodes of week 1 become orange nodes in week 10 , and the yellow nodes of week 1 are colored light blue in week 10. Position 2 was part of the giant component in week 1, and is more strongly connected to it in week 10 . The students in position 4 in week 1 were separate from the giant component, but connected to it (now as position 2) in week 10. Positions 1, 3, and 5 are less stable between the two time points.

In the ISLE alluvial diagram, position 1 keeps three of its original four students and adds two more. Interestingly, in week 1 none of the position 1 nodes reported any links to each other, but in week 10 the same students are well connected. In general, positions in this section showed more mixing than stability in the time between surveys. This is likely due in part to the small section size, which means that even two students shifting substantially changes the block membership.

In the alluvial diagram for context-rich problems, blocks 2 and 4 keep substantial fractions of their membership together (though the largest coherent group in 4 is relabeled to position 3 in the week 10 results). Blocks 1,3 , and 5 show a fair amount of splitting after week 1 . The week 10 survey had a response rate of $42 \%$, so it is possible that the block assignments and stability picture would change with more complete student link data.

Figure 7 suggests that for many students, their initial block assignment does little to predict their week 10 position. This is not particularly surprising, as the first survey happens early in the class, when they have generally fewer connections and may not keep all of those initial links. One point of interest is the tendency for stable blocks to accumulate in the giant component. This appeared to happen in the Peer Instruction and SCALE-UP sections, but should be checked for in a wider sample of data, preferably with response rates of $70 \%$ or higher. This might indicate students who already had peer groups coming in to the class, or alternately students who "found their people" early. Whatever the cause, these relatively stable social groups also became more central in the whole-class network. 


\section{G. Interpreting blockmodels}

Blockmodels can be interpreted by validating against actor attributes, by describing the overall blockmodel, and by detailing the individual positions [27]. Detailed actor information is not available for most of our data, but might include demographic details, grades or other learning outcomes, or schedule information such as students' lab sections [20]. We can describe the overall blockmodel and individual positions even without additional data.

\section{Describing the blockmodel}

A large-scale view of the network structure can come from the image matrix, which summarizes the blockmodel as an adjacency matrix of 1's and 0's between and within blocks. The reduced networks in Figs. 3-6 show the image matrix visually. Blocks are thresholded to 0 or 1 by comparing their internal density values to a single value $(\alpha)$ for the network. We use the density of the full network as the threshold value $\alpha$ [27]. For example, a three-person block would have six possible ties among members, and a density of 0.5 if three of those ties were actually present. A network density of $\alpha=0.1$ would mean that the three-person block had an image matrix value of 1 for its self-tie.

Image matrices can be compared to ideal types as a limiting case. Ideal patterns include cohesive subgroups, center and periphery, centralized, and others [27]. In the cohesive subgroups pattern, actors in one block primarily talk to each other and not to members of other blocks, corresponding to an image matrix with 1's on the diagonal and 0's elsewhere:

$$
\left[\begin{array}{llll}
1 & 0 & 0 & 0 \\
0 & 1 & 0 & 0 \\
0 & 0 & 1 & 0 \\
0 & 0 & 0 & 1
\end{array}\right]
$$

In a centralized network, all positions link to one other position, whose members also talk among themselves:

$$
\left[\begin{array}{llll}
1 & 0 & 0 & 0 \\
1 & 0 & 0 & 0 \\
1 & 0 & 0 & 0 \\
1 & 0 & 0 & 0
\end{array}\right]
$$

Reference [27] (Chap. 10) and Ref. [29] discuss more detailed examples.

For comparison, the week 1 image matrices for each of the sections in this paper are shown below:

$$
\begin{aligned}
\text { PI } & =\left[\begin{array}{lllll}
1 & 1 & 0 & 0 & 0 \\
0 & 1 & 0 & 0 & 0 \\
0 & 0 & 1 & 0 & 0 \\
0 & 0 & 1 & 1 & 0 \\
0 & 0 & 0 & 0 & 0
\end{array}\right] \\
\text { SCALE - UP } & =\left[\begin{array}{lllll}
1 & 0 & 0 & 0 & 0 \\
1 & 1 & 0 & 0 & 0 \\
0 & 0 & 1 & 0 & 0 \\
0 & 0 & 0 & 1 & 0 \\
0 & 0 & 0 & 0 & 0
\end{array}\right], \\
\text { ISLE } & =\left[\begin{array}{lllll}
0 & 1 & 0 & 0 & 0 \\
1 & 1 & 0 & 0 & 0 \\
0 & 0 & 1 & 0 & 0 \\
0 & 0 & 0 & 1 & 0 \\
0 & 0 & 0 & 0 & 0
\end{array}\right] \\
\text { Context rich } & =\left[\begin{array}{lllll}
1 & 1 & 0 & 0 & 0 \\
1 & 0 & 0 & 0 & 0 \\
0 & 0 & 1 & 0 & 0 \\
0 & 1 & 0 & 1 & 0 \\
0 & 0 & 0 & 0 & 0
\end{array}\right]
\end{aligned}
$$

and the week 10 image matrices are

$$
\begin{aligned}
\mathrm{PI} & =\left[\begin{array}{lllll}
1 & 0 & 0 & 0 & 0 \\
1 & 1 & 0 & 0 & 0 \\
0 & 0 & 1 & 0 & 0 \\
0 & 0 & 0 & 1 & 0 \\
0 & 0 & 0 & 0 & 0
\end{array}\right] \\
\text { SCALE }-\mathrm{UP} & =\left[\begin{array}{llll}
1 & 0 & 0 & 0 \\
0 & 1 & 0 & 0 \\
0 & 0 & 1 & 0 \\
0 & 0 & 0 & 1
\end{array}\right],
\end{aligned}
$$

$$
\text { ISLE }=\left[\begin{array}{llll}
1 & 0 & 0 & 0 \\
0 & 1 & 0 & 0 \\
0 & 0 & 1 & 0 \\
0 & 0 & 0 & 1
\end{array}\right] \text { Context rich }=\left[\begin{array}{llll}
0 & 0 & 0 & 0 \\
1 & 1 & 1 & 0 \\
0 & 0 & 1 & 0 \\
0 & 0 & 0 & 1
\end{array}\right] \text {. }
$$

Many of the class networks do not perfectly match any of the ideal types, though the alignment is generally closer in week 10. In two cases-late-semester SCALE-UP and ISLE - the cohesive subgroups pattern is exactly present. More commonly, the image matrices largely resemble cohesive subgroups, with one or two links "extra" or a 
self-tie missing. The image matrices for context-rich problems display the least resemblance to any of the ideal types, but also had the lowest response rates. It is possible that a more regular structure was simply not observed.

\section{Describing individual positions}

Each position in the blockmodel can also be considered in light of how it connects to the others and its ratio of communication inside vs outside the block [27] (Chap. 10). If there are $N$ nodes in the network, and $N_{k}$ in position $k$, there are $N_{k}\left(N_{k}-1\right)$ ties possible within the position. Collectively, the position has $N_{k}(N-1)$ ties possible with the whole network (including itself). We can quantify a block's tendency toward self-interactions by comparing to the ratio of internal to total ties. For blocks with no internal vs external preference, this ratio is

$$
\frac{N_{k}\left(N_{k}-1\right)}{N_{k}(N-1)}=\frac{N_{k}-1}{N-1} \text {. }
$$

Positions with a greater ratio of internal:total ties than this value prefer to communicate among themselves. Positions with a smaller fraction of internal:total ties than Eq. (3) prefer to communicate "outward." By also considering whether the position tends to receive ties (proportion received $\sim 0$ or $>0$ ), a social function for the group can be approximated. Burt [28] names these roles as

- Isolate (prefer internal ties, $\sim 0$ received),

- Primary (prefer internal, $>0$ received),

- Sycophant (prefer external, $\sim 0$ received), and

- Broker (prefer external, $>0$ received).

"Sycophant" is a pointlessly negative term in class settings where students are encouraged to seek help from peers, so that label is worth revisiting, but this position profile does not appear in our data.

When we calculate the tie ratios and received links for each position in the week 1 and week 10 networks, we find that the island positions shown on many of the reduced networks are actually a mixture of primary and isolate types. [For true isolates-dark blue nodes on the socigramsthe ratio to compare to Eq. (3) is undefined because there are no ties, internal or otherwise. We include them with the isolate classification.] The results by class type were as follows:

- Peer Instruction: Both week 1 and week 10 have four primary positions (preferring within-block interactions, incoming ties) and one isolate.

- SCALE-UP: Week 1 has one primary (block 1) and four isolates (blocks 2-5). Week 10 has four primaries.

- ISLE: Week 1 has one broker (incoming ties but low internal communication), one primary, and three isolates, in that order. Week 10 has four isolates (blocks 1 and 2 have only one incoming link each).

- Context-rich problems: Week 1 has one primary, one broker, and three isolates. Week 10 has four primaries.
Figure 9 in the Supplemental Material [41] shows the link ratios and counts of incoming links for each position.

Considering tie ratios and incoming links adds nuance to the reduced network plots (Figs. 3-6). In many cases, the number of incoming ties was small enough to be thresholded out of the reduced network display, but is not actually zero. In the SCALE-UP section, an early-semester trend of isolate positions became a late-semester tendency for primary positions, reflecting a higher level of overall connectivity even as subgroups gained coherence. On the other hand, for ISLE, the late-semester islands on the reduced network have very little between-position communication. The context-rich problems section, despite its many partially or completely unobserved ties, has a large amount of between-position traffic.

\section{DISCUSSION}

The results of positional analysis live between understanding individuals and the class as a whole. It allows us to hypothesize about the ways the enactment of a pedagogy influences the "terrain" of a class. While we can make inferences about the enactment of the pedagogy in these instances, it is impossible to tease out (at least from a single institution) how the design of the pedagogy and the enactment differ.

\section{A. Block structure and reduced networks}

The mixture of isolate and primary positions, which can both appear as self-connected islands on reduced networks, is interesting. The distinction between isolates and primaries is not trivial-some positional analysis approaches argue that any link at all between blocks should be regarded as a positive tie on the image matrix, and a complete absence of links is the most significant structural cue to look for [29]. If this stricter standard were to be applied, the reduced networks would be more interlinked, and only a small number of self-linked islands would remain.

Reduced networks are a representation that is uncommon outside of positional analysis, though there are some similar ideas in the flow diagrams of the Infomap algorithm [25] or in node-consolidating analyses of large networks [55]. Reduced networks abstract out most of the detail of the full network diagram, but permit comparing position structure between networks of different sizes. We also found that the reduced networks were relatively stable to missing data (details in Supplemental Material [41]). We would not recommend reduced networks as the only description of a network, because the nuance they lose is important to many research questions. But in our data, they show broad structural similarities between classroom networks of different sizes and densities. A wider future data collection, including multiple sites implementing the same curriculum, would allow for claims about what 
block-level structures are "typical" for active learning environments.

\section{B. Curricular impacts on positions}

The two most common types of positions across the different curricula were isolate and primary, both of which prefer internal links. It should also be noted that the collection of students that make up these positions are not equivalent to classroom groups. For example, in SCALE-UP one might expect to find positions that include the nine students that make up a table of students as prescribed by the SCALE-UP approach. Instead we find that the whole class network is dissociable into four separate components, each of which includes a number of students much larger than nine. This is not simply an artifact of the number of CONCOR splits, because particularly the late-term positions are not made up of isolated "pods," but consist of well-linked parts of the giant component. We do not have data on the table assignment of students within the SCALE-UP class, but we can infer that the prescribed tables are interacting, plausibly with other adjacent tables of students. The positions identified in SCALE-UP late in the term might indicate that several groups of tables develop working relationships with one another and these relationships become more productive over time, resulting in blocks where there is a preference for internal interaction but still some interaction with other blocks. Similar patterns can be seen in this enactment of the ISLE pedagogy. The week 10 context-rich problem solving environment yielded entirely primary positions, as well as a high average degree in the network despite the sizable fraction of missing data. This indicates that in this enactment, students are building many connections, possibly through the sharing of problem solving approaches.

In addition to the prevalence of primary and isolate positions, the lack of brokering and sycophant positions is interesting. The broker position provides a bridge to connect disjoint groups. In active learning pedagogies, it seems unlikely to find groups of students in this role; individuals may play such a role [56], but groups of students would be less likely to connect other groups. The lack of the sycophant position suggests that there are not large groups of students looking to gain from other groups in an unreciprocated way. This suggests that across the active learning we studied, individual students who needed help were able to identify members within their own block who could provide this help.

\section{CONCOR and community detection}

In Sec. I, we noted that CONCOR can be classified as a community detection algorithm, because it seeks to partition a network based only on information contained in the network object. By consulting the network diagrams in Figs. 3-6, we can now appreciate some of the differences between positional analysis and other community detection methods. In Fig. 3(a), for example, the week 1 diagram shows that block 3 (green nodes) consists of several isolated components and a subset of the nodes in the giant component. This particular collection of nodes would not be partitioned together by methods such as Infomap or edge betweenness, because community detection algorithms do not typically group entirely disconnected components into the same partition. However, CONCOR's criterion is linking behavior, not direct connection. The separate small components in block 3 all show a similar "stringy" structure of short chains with low transitivity and low reciprocity. The green nodes in the giant component have a similar trend of low-reciprocity, low-transitivity links, and this trend is different from the more interlinked parts of the giant component (yellow, orange, and light blue nodes). Other community detection methods [17] address questions such as "which students work closely together?" but CONCOR targets the complementary question of "which students have similar connection-building behavior?" To characterize the social texture of an active learning classroom, both perspectives are useful.

\section{CONCLUSIONS}

\section{A. Research questions}

The goal of this investigation was to compare the network positions available in four active learning classroom types using the method of positional analysis. This technique provides a kind of mesoscale description of social structure, between fine-grained dyad interactions and whole-network descriptions. The investigation was structured around three research questions, discussed below:

1. What network positions emerge from the four different curriculum types? As detailed in Sec. III G 2 and Fig. 6 of the Supplemental Material [41], most of the node blocks identified by CONCOR preferred links among themselves, but possessed at least some incoming links, leading to a mixture of isolate and primary positions. The image matrices $(0 / 1$ representations of the reduced networks) showed a corresponding tendency toward the "coherent subgroups" type, where most positions connected only to themselves.

2. What differences exist between early-and late-term network positions? The week 1 networks had more deviations from the pattern described above, while the week 10 blockmodel plots generally showed more distinct and coherent positions. Link reciprocity also increased from early to late in the term, as did the density and average degree (though not always significantly, see Table II). This increase in network connectivity is likely a mixture of effects: social connections forming through the class, plus more students knowing each other's names by the time of the late-semester network survey. 
3. What major similarities or differences exist in network positions across learning environments? At the level of analysis provided by CONCOR, there appear to be more similarities than differences in the network position structure. From the data available here, we might say that the "signature" of active learning at the positional analysis level is one of coherent subgroups plus a handful of interposition links. Only a small number of students, if any, are true isolates (degree 0 ).

\section{B. Significance to PER}

Active learning classrooms often encourage students to work in small groups or pairs, so it may be unsurprising that the class-level network structure most closely resembles coherent subgroups. However, many social groups of this size show different positional patterns. Classic blockmodeling studies often find unofficial structures of authority, deference, or a center and periphery structure between positions [29]. CONCOR studies of the world trade network have explored its core-periphery structure [57], reinforcing that the coherent subgroups pattern is not simply an artifact of the algorithm. If a block of students was systematically more popular under the "meaningful interaction" prompt, in an unreciprocated way, the reduced networks would show a more starlike structure with singledirection links going to a central hub. In comparison to those findings, the social structure of the classes we surveyed appears to be "flatter," with less hierarchical tendency for the ties between positions.

Positional analysis provides a different lens for examining networks: more detail than whole-network statistics like average degree or centralization, but abstracting away some of the node-level detail of centrality scores. If centrality analyses ask "who has the most power?", positional analyses ask "what is the terrain?" Both characterizations are valuable for understanding the complex system of interactions in a classroom.

One thing that is not obvious from this analysis is whether there is a preferred or ideal position structure for an active learning environment. Anecdotally, when discussing network analysis with instructors for the first time, a common expectation is that "everyone will just work with the smart student." If it were the case that students informally identified the student(s) most likely to know the correct answers, and worked with those people exclusively, we might see a hierarchy pattern on the image matrix: a few students occupying a position that all other positions directed links toward, creating a single column and row with 1's and 0's in most or all other spaces. This pattern, which did not appear in our data, might indicate an inefficient use of the opportunities in group collaboration. While a few students will often have more prior knowledge and practice with a topic, even initially high-scoring students benefit from collaborating [51], so a strong hierarchy is unlikely to be a desirable signature for active learning environments. Beyond that, other factors are likely to intervene, such as the physical layout of the classroom and the social structure encouraged by the curriculum. For example, Peer Instruction directs students to talk to a neighbor, and in a large lecture hall with immobile seating, this may tend to lead to "chains" in the network rather than large interconnected clusters [42]. The coherent subgroups pattern here, when checked against the network diagrams, often "unpacks" into a collection of higher-density groups with a smaller number of ties between them. For this study, we chose project sites with expert implementations of successful active learning curricula, so we argue that the coherent subgroups pattern is one (though not necessarily the only) signature of a well-functioning active learning environment.

\section{LIMITATIONS AND FUTURE WORK}

One of the substantial challenges of this study was response rate: even though instructors endorsed the survey data being collected in their classes, the invitation to participate came from an outside researcher, and this may have contributed to the lower response in many sections. The highest-response section in the data was one where the instructor allocated a few minutes of class time for students to do the survey, but we did not ask for this broadly to keep the "cost" to instructors low. Ideally, structural analyses of directed networks would use data with a response rate of $70 \%$ or higher, which was unfeasible in our sample. We were able to somewhat check for the severity of this effect by comparing the all-response with complete cases data (see Supplemental Material [41]), but better estimates ultimately must come from more complete sampling of the network. Instructors collecting data in their own classes tend to see higher return rates, so we hope that other researchers may take up this analysis method for their own classrooms.

Data from a wider range of classrooms can also help to separate out the design of a pedagogy from its enactment. Even widely known and well-documented pedagogies such as Peer Instruction can vary greatly in enactment [58], and we expect that such implementation differences could have consequences for the student networks that arise. The $\mathrm{R}$ code we developed for the CONCOR algorithm is publicly available [35], including blockmodels, reduced networks, and image matrices. We anticipate that the coherent subgroups pattern we saw in many cases may be common across a wider range of active learning physics classes.

Unlike many other PER analyses of student networks mentioned in Sec. I, this study did not include outcome data to link with network properties. We did find that student networks generally become more connected over the course of the semester, as noted in other work [14,15]. Combining positional analysis with outcome or demographic data opens additional possibilities. Outcome information such 
as pass or fail rates, concept inventory scores, or other measures of learning would allow researchers to test whether a more stable initial block assignment is associated with more favorable grade outcomes (i.e., if students who "find a home" in the network sooner are more successful in the class). Alternately, the coherent subgroups pattern appears to be common but not ubiquitous, so outcomes could be compared between students in isolate and primary positions, or between more and less popular primary positions (the most-popular positions being those with an additional incoming link on the reduced network, such as block 1 in the week 10 Peer Instruction network). Demographic data such as gender or race and ethnicity might reveal additional nuances in the position structure. Node-level information about student demographics would allow looking for effects such as homophily, the tendency to socially group with others we perceive as "like" us [20,59], or looking for widespread disparities in who sends and receives links.

Finally, networks with multirelation or longitudinal data are especially good candidates for positional analysis.
By combining snapshots of the connections between nodes and the pattern of connection between positions, more complicated patterns of interlocking roles can be extracted $[27,29]$. PER studies with access to such data $[10,11]$ might benefit from this additional layer of analysis.

By now, there are a number of descriptive results of student networks in introductory physics courses. To move beyond this stage of collecting baselines and into more inferential and predictive questions, network analysis in PER needs careful survey design, reasonably standard data collection protocols, and community discussion about the models and measures most appropriate to capture the interactions of active learning.

\section{ACKNOWLEDGMENTS}

We are grateful to the instructors who allowed us to observe and survey their courses. This work was supported in part by the National Science Foundation under Grants No. DUE-1711017 and No. DUE-1712341.
[1] S. Freeman, S. L. Eddy, M. McDonough, M. K. Smith, N. Okoroafor, H. Jordt, and M. P. Wenderoth, Active learning increases student performance in science, engineering, and mathematics, Proc. Natl. Acad. Sci. U.S.A. 111, 8410 (2014).

[2] E. J. Mastascusa, W. J. Snyder, and B. S. Hoyt, Effective Instruction for STEM Disciplines: From Learning Theory to College Teaching (Jossey-Bass, Indianapolis, IN, 2011).

[3] D. E. Meltzer and R. K. Thornton, Resource letter ALIP-1: Active-learning instruction in physics, Am. J. Phys. 80, 478 (2012).

[4] E. Brewe, The roles of engagement: Network analysis in physics education research, in Getting Started in Physics Education Research, Reviews in PER, Vol. 2, edited by C. Henderson and K. A. Harper (American Association of Physics Teachers, College Park, MD, 2018).

[5] D. Z. Grunspan, B. L. Wiggins, and S. M. Goodreau, Understanding classrooms through social network analysis: A primer for social network analysis in education research, CBE Life Sci. Educ. 13, 167 (2014).

[6] E. Brewe, L. Kramer, and V. Sawtelle, Investigating student communities with network analysis of interactions in a physics learning center, Phys. Rev. ST Phys. Educ. Res. 8, 010101 (2012).

[7] M. Bodin, Mapping university students' epistemic framing of computational physics using network analysis, Phys. Rev. ST Phys. Educ. Res. 8, 010115 (2012).

[8] J. Bruun, M. Lindahl, and C. Linder, Network analysis and qualitative discourse analysis of a classroom group discussion, Int. J. Res. Method Educ. 42, 317 (2019).
[9] A. Traxler, A. Gavrin, and R. Lindell, Networks identify productive forum discussions, Phys. Rev. Phys. Educ. Res. 14, 020107 (2018).

[10] J. Bruun and E. Brewe, Talking and learning physics: Predicting future grades from network measures and Force Concept Inventory pretest scores, Phys. Rev. ST Phys. Educ. Res. 9, 020109 (2013).

[11] D. L. Vargas, A. M. Bridgeman, D. R. Schmidt, P. B. Kohl, B. R. Wilcox, and L. D. Carr, Correlation between student collaboration network centrality and academic performance, Phys. Rev. Phys. Educ. Res. 14, 020112 (2018).

[12] J. P. Zwolak, M. Zwolak, and E. Brewe, Educational commitment and social networking: The power of informal networks, Phys. Rev. Phys. Educ. Res. 14, 010131 (2018).

[13] J. Forsman, C. Linder, R. Moll, D. Fraser, and S. Andersson, A new approach to modelling student retention through an application of complexity thinking, Studies Higher Educ. 39, 68 (2014).

[14] E. Brewe, L. H. Kramer, and G. E. O'Brien, Changing participation through formation of student learning communities, AIP Conf. Proc. 1289, 85 (2010).

[15] A. Traxler, Community structure in introductory physics course networks, in Proceedings of the 2015 Physics Education Research Conference, College Park, MD, edited by A.D. Churukian, D. L. Jones, and L. Ding (AIP, New York, 2015), pp. 331-334, https://doi.org/ 10.1119/perc.2015.pr.078.

[16] A. K. Dey, Y. R. Gel, and H. V. Poor, What network motifs tell us about resilience and reliability of complex networks, Proc. Natl. Acad. Sci. U.S.A. 116, 19368 (2019).

[17] S. Fortunato, Community detection in graphs, Phys. Rep. 486, 75 (2010). 
[18] E. Brewe, J. Bruun, and I. Bearden, Using module analysis for multiple choice responses: A new method applied to Force Concept Inventory data, Phys. Rev. Phys. Educ. Res. 12, 020131 (2016).

[19] J. Wells, R. Henderson, A. Traxler, P. Miller, and J. Stewart, Exploring the structure of misconceptions in the Force and Motion Conceptual Evaluation with Modified Module Analysis, Phys. Rev. Phys. Educ. Res. 16, 010121 (2020).

[20] J. Bruun and I. G. Bearden, Time development in the early history of social networks: Link stabilization, group dynamics, and segregation, PLoS One 9, e112775 (2014).

[21] M. E. J. Newman, The structure and function of complex networks, SIAM Rev. 45, 167 (2003).

[22] M. E. J. Newman, Modularity and community structure in networks, Proc. Natl. Acad. Sci. U.S.A. 103, 8577 (2006).

[23] K. A. Frank, Identifying cohesive subgroups, Soc. Networks 17, 27 (1995).

[24] M. Girvan and M. E. J. Newman, Community structure in social and biological networks, Proc. Natl. Acad. Sci. U.S.A. 99, 7821 (2002).

[25] M. Rosvall and C. T. Bergstrom, Maps of random walks on complex networks reveal community structure, Proc. Natl. Acad. Sci. U.S.A. 105, 1118 (2008).

[26] J. Scott, Social physics and social networks, in The SAGE Handbook of Social Network Analysis, edited by J. Scott and P. J. Carrington (SAGE Publications, Thousand Oaks, CA, 2011), pp. 55-66.

[27] S. Wasserman and K. Faust, Social Network Analysis: Methods and Applications, Structural analysis in the social sciences (Cambridge University Press, New York, NY, 1994).

[28] R. S. Burt, Positions in networks, Social Forces 55, 93 (1976).

[29] H. C. White, S. A. Boorman, and R. L. Breiger, Social structure from multiple networks. I. Blockmodels of roles and positions, Am. J. Sociology 81, 730 (1976).

[30] R. L. Breiger, S. A. Boorman, and P. Arabie, An algorithm for clustering relational data with applications to social network analysis and comparison with multidimensional scaling, J. Math. Psychol. 12, 328 (1975).

[31] A. Ferligoj, P. Doreian, and V. Batagelj, Positions and roles, in The SAGE Handbook of Social Network Analysis, edited by J. Scott and P. J. Carrington (SAGE Publications, Thousand Oaks, CA, 2011), pp. 434-446.

[32] A. Žnidaršič, A. Ferligoj, and P. Doreian, Non-response in social networks: The impact of different non-response treatments on the stability of blockmodels, Soc. Networks 34, 438 (2012).

[33] R Core Team, R: A Language and Environment for Statistical Computing (R Foundation for Statistical Computing, Vienna, Austria 2017).

[34] A. Slez, concoR: CONvergence of Iterated CORrelations (2015), https://rdrr.io/github/aslez/concoR/.

[35] T. Suda and A. Traxler, concorR: CONCOR and Supplemental Functions (2020), https://cran.r-project.org/package= concorR.

[36] R. S. Burt, A note on missing network data in the general social survey, Soc. Networks 9, 63 (1987).
[37] J. A. Smith, J. Moody, and J. H. Morgan, Network sampling coverage II: The effect of non-random missing data on network measurement, Soc. Networks 48, 78 (2017).

[38] J. A. Smith and J. Moody, Structural effects of network sampling coverage I: Nodes missing at random, Soc. Networks 35, 652 (2013).

[39] M. Huisman, Imputation of missing network data: Some simple procedures, J. Social Struct. 10, 1 (2009).

[40] D. Stork and W. D. Richards, Nonrespondents in communication network studies: Problems and possibilities, Group Organ. Manage. 17, 193 (1992).

[41] See Supplemental Material at http://link.aps.org/ supplemental/10.1103/PhysRevPhysEducRes.16.020129 for respondents-only network results and link ratio calculations for positions.

[42] K. Commeford, E. Brewe, and A. Traxler, Characterizing active learning environments in physics using network analysis and COPUS observations, arXiv:2008.05325.

[43] P. V. Marsden, Survey methods for network data, in The SAGE Handbook of Social Network Analysis, edited by J. Scott and P. J. Carrington (SAGE Publications, Thousand Oaks, CA, 2011), pp. 370-388.

[44] E. Mazur, Peer Instruction: A User's Manual (Prentice Hall, Upper Saddle River, NJ, 1997).

[45] C. H. Crouch, J. Watkins, A. P. Fagen, and E. Mazur, Peer Instruction: Engaging students one-on-one, all at once, in Research-Based Reform of University Physics, Reviews in Physics Education Research, Vol. 1, edited by E. F. Redish and P.J. Cooney (American Association of Physics Teachers, College Park, MD, 2007).

[46] C. Henderson, Promoting instructional change in new faculty: An evaluation of the physics and astronomy new faculty workshop, Am. J. Phys. 76, 179 (2008).

[47] R. J. Beichner, J. M. Saul, D. S. Abbott, J. J. Morse, D. L. Deardorff, R. J. Allain, S. W. Bonham, M. H. Dancy, and J.S. Risley, The Student-Centered Activities for Large Enrollment Undergraduate Programs (SCALE-UP) project, in Research-Based Reform of University Physics, Reviews in Physics Education Research, Vol. 1, edited by E. F. Redish and P. J. Cooney (American Association of Physics Teachers, College Park, MD, 2007).

[48] E. Etkina and A. Van Heuvelen, Investigative Science Learning Environment-A science process approach to learning physics, in Research-Based Reform of University Physics, Reviews in Physics Education Research, Vol. 1, edited by E. F. Redish and P. J. Cooney (American Association of Physics Teachers, College Park, MD, 2007).

[49] E. Frodermann (maintainer), Minnesota Model for Large Introductory Courses (2014), http://groups.physics.umn .edu/physed/Research/MNModel/MMt.html.

[50] K. Heller and P. Heller, Cooperative Problem Solving in Physics: A User's Manual (2010), https://www.aapt.org/ Conferences/newfaculty/upload/Coop-Problem-SolvingGuide.pdf.

[51] P. Heller, R. Keith, and S. Anderson, Teaching problem solving through cooperative grouping. Part 1: Group versus individual problem solving, Am. J. Phys. 60, 627 (1992). 
[52] G. Csardi and T. Nepusz, The igraph software package for complex network research, InterJournal Complex Systems, 1695 (2006), http://www.interjournal.org/ manuscript_abstract.php?361100992.

[53] T. A. B. Snijders and S. P. Borgatti, Non-parametric standard errors and tests for network statistics, Connections 22, 61 (1999), https://assets.noviams.com/novi-file-uploads/insna/ Connections_Archive/1999_Volume_22__Issue_2.pdf.

[54] M. Rosvall and C. T. Bergstrom, Mapping change in large networks, PLoS One 5, e8694 (2010).

[55] N. Stanley, R. Kwitt, M. Niethammer, and P. J. Mucha, Compressing networks with super nodes, Sci. Rep. 8, 10892 (2018).

[56] J. Pulgar, C. Candia, and P. M. Leonardi, Social networks and academic performance in physics:
Undergraduate cooperation enhances ill-structured problem elaboration and inhibits well-structured problem solving, Phys. Rev. Phys. Educ. Res. 16, 010137 (2020).

[57] W. Luo, P. Yin, Q. Di, F. Hardisty, and A. M. MacEachren, A geovisual analytic approach to understanding geo-social relationships in the international trade network, PLoS One 9, e88666 (2014).

[58] C. Turpen and N.D. Finkelstein, Not all interactive engagement is the same: Variations in physics professors' implementation of Peer Instruction, Phys. Rev. ST Phys. Educ. Res. 5, 020101 (2009).

[59] M. McPherson, L. Smith-Lovin, and J. M. Cook, Birds of a feather: Homophily in social networks, Annu. Rev. Sociol. 27, 415 (2001). 